\title{
VERY LOW-LUMINOSITY OBJECTS IN STAR-FORMING REGIONS
}

\author{
MOTOHIDE TAMURA \\ National Astronomical Observatory of Japan, Osawa 2-21-1, Mitaka, Tokyo 181, Japan \\ YOICHI ITOH AND YUMIKO OASA \\ University of Tokyo, Osawa 2-21-1, Mitaka, Tokyo 181, Japan \\ ALAN TOKUNAGA \\ Institute for Astronomy, University of Hawaii, 2680 Woodlawn Drive, Honolulu, HI96822, \\ $U S A$ \\ AND \\ KOJI SUGITANI \\ Nagoya City University, Mizuho-ku, Nagoya 467, Japan
}

\begin{abstract}
In order to tackle the problems of low-mass end of the initial mass function (IMF) in star-forming regions and the formation mechanisms of brown dwarfs, we have conducted deep infrared surveys of nearby molecular clouds. We have found a significant population of very lowluminosity sources with IR excesses in the Taurus cloud and the Chamaeleon cloud core regions whose extinction corrected $\mathrm{J}$ magnitudes are 3 to $8 \mathrm{mag}$ fainter than those of typical $\mathrm{T}$ Tauri stars in the same cloud. Some of them are associated with even fainter companions. Follow-up IR spectroscopy has confirmed for the selected sources that their photospheric temperature is around 2000 to $3000 \mathrm{~K}$. Thus, these very low-luminosity young stellar sources are most likely very low-mass $\mathrm{T}$ Tauri stars, and some of them might even be young brown dwarfs.
\end{abstract}

\section{Introduction}

One of the most important issues about the IMF is its slope, particularly at its lower-mass end extending towards substellar region. In addition, whether it generally turns over and whether its slope differs among various star-forming regions are important questions to be explored. This issue is directly related with the formation mechanisms of brown dwarfs. A variety of techniques at various wavelengths such as X-ray, optical, and infrared surveys have been applied to search for low-mass objects in many star-forming regions, but infrared studies have two advantages. First, lowmass objects in regions of star-formation will be in the contraction phase and radiate their energy mostly at infrared wavelengths. Second, at infrared wavelengths, the radiation from the sources is less affected by the cloud and circumstellar matter extinction than at optical wavelengths. In addition, recent great advances in infrared array detectors enable us to conduct deep and wide-area near-infrared surveys. Armed with these advantages, we have conducted deep near-infrared surveys of nearby molecular clouds of Taurus, Chamaeleon, and Perseus. In this contribution, we report on the results of the surveys of the Taurus and Chamaeleon dark clouds, which resulted in the discovery of a number of very low-luminosity young stellar objects (YSOs), and the results of our follow-up spectroscopy and high-resolution imaging of the newly detected very low-mass YSOs.

\section{Observations and Results}

We have conducted a JHK survey of a part of the Taurus molecular cloud complex with a completeness limit of $\mathrm{J}=15.2 \mathrm{mag}$ (an area of 1.5 square degrees covering most of the Heiles Cloud 2). We have detected a number of low-luminosity sources with near-infrared excesses on the J-H/H-K diagram, which we ascribe to the emission from the circumstellar matter similar to that of optical $T$ Tauris stars (TTSs). However, their luminosity is 3 to 6 mag fainter than those of typical TTSs 
in Taurus. These sources are too faint to be detected with the previous YSO searches including optical $\mathrm{H} \alpha, I R A S$, and X-ray surveys. Their masses estimated from the combination of the J-band fluxes and the evolutionary tracks for low-mass YSOs (D'Antona \& Mazzitelli 1994), assuming ages of typical TTSs in the cloud, are near the stellar/substellar boundary and some of them are indeed in the substellar region. The luminosity function of these low-luminosity YSOs does not appear to have any cut-off at its low-end. See Itoh, Tamura, \& Gatley (1996) for the details of this observation.

In the core region of the Chamaeleon molecular cloud I complex, we have conducted a highersensitivity JHK survey of the cloud core. With a completeness limit of $J=17.1 \mathrm{mag}$, we have identified 13 YSO candidates within the central 30 square arcminutes. Some of them are of very low-luminosity, even 8 mag fainter than the typical TTSs in the same cloud. The luminosity function of the YSOs seems to be bimodal in this region, and the fainter group of YSOs shows an increase of counts toward the detection limit (Oasa, Tamura, \& Sugitani 1997).

\section{Follow-Up Observations and Discussion}

The photometric approach described above is very useful to identify the very low-luminosity YSOs with IR excesses. However, in order to correctly estimate the mass of the detected YSOs, it is necessary estimate the age of the sources. If the age of the sources is older than our assumed ages of typical TTSs, the low-luminosity of the detected could be explained by their older ages. One of the best ways for determining both masses and ages simultaneously is determining the effective temperature and luminosity of the sources, then placing them in the HR-diagram and comparing with the theoretical YSO tracks. In order to determine the effective temperature, we have conducted near-infrared spectroscopy of the selected low-luminosity sources detected in our Taurus survey with CGS4 at the 3.8-m UKIRT and with OASIS at the Okayama 1.88-m telescope (Itoh, Tamura, \& Tokunaga 1997). First, we have confirmed that most of our low-luminosity sources are not simple background giants/dwarfs and their spectra are more or less similar to those of TTSs. We have then used the $\mathrm{Na} / \mathrm{Ca}$ line ratio to determine that their photospheric temperature is around 2000 to 3000 $\mathrm{K}$. The results as well as the photometric results are therefore consistent with the interpretation that the very low-luminosity YSOs detected in our near-infrared surveys are very low-mass TTSs and that some of them might even be young brown dwarfs.

We have also been conducting high-resolution imaging of the detected low-luminosity YSOs and have discovered that some sources appear to be associated with even fainter companions. The companions also have IR excesses. If these two sources have the same age, the fainter companion is less massive than the primary low-luminosity YSO, which is strong evidence that the nearby star-forming region is the site of brown dwarf formation. Brown dwarfs appear to form in both isolated mode and as companions, although the relative frequencies are not clear. Both extensive deep and wide area surveys and high-resolution observations will be necessary for a more statistical study. The simultaneous-band infrared imager for unbiased surveys (SIRIUS) and the infrared coronagraphic imager with adaptive optics (CIAO; Tamura et al. 1997) for the Japanese Subaru 8-m telescope, both now under development, will be a most powerful tool for this study.

\section{References}

D'Antona, F., \& Mazzitelli, I. 1994, ApJS, 164, 349

Itoh, Y., Tamura, M., \& Gatley, I. 1996, ApJ, 465, L129

Itoh, Y., Tamura, M., \& Tokunaga, A. 1997, in preparation

Oasa, Y., Tamura, M., \& Sugitani, K. 1997, in preparation

Tamura, M. et al. 1997, in Extrasolar planets and brown dwarfs, ed. R. Rebolo, ASP Conf Ser., in press 